\title{
Mitochondrial Diseases in Man and Mouse
}

\author{
Douglas C. Wallace
}

\begin{abstract}
Over the past 10 years, mitochondrial defects have been implicated in a wide variety of degenerative diseases, aging, and cancer. Studies on patients with these diseases have revealed much about the complexities of mitochondrial genetics, which involves an interplay between mutations in the mitochondrial and nuclear genomes. However, the pathophysiology of mitochondrial diseases has remained perplexing. The essential role of mitochondrial oxidative phosphorylation in cellular energy production, the generation of reactive oxygen species, and the initiation of apoptosis has suggested a number of novel mechanisms for mitochondrial pathology. The importance and interrelationship of these functions are now being studied in mouse models of mitochondrial disease.
\end{abstract}

Mitochondrial defects occur in a wide variety of degenerative diseases, aging, and cancer. One of the first indications that mitochondria may play a role in pathogenesis was the report nearly 40 years ago of a patient with hypermetabolism whose skeletal muscle contained large numbers of abnormal mitochondria, a condition now known as mitochondrial myopathy (Fig. 1) (1). It is now clear that mitochondrial diseases encompass an extraordinary assemblage of clinical problems, commonly involving tissues that have high energy requirements such as heart, muscle, and the renal and endocrine systems. The genetic and molecular complexities of these diseases, which typically display a bewildering array of inheritance patterns, have been studied intensively over the past decade (2-5).

The first mitochondrial diseases to be understood at the molecular level were the maternally inherited Leber's hereditary optic neuropathy (LHON), a sudden-onset blindness resulting from a mitochondrial DNA (mtDNA) missense mutation (6), and a spontaneously occurring group of neuromuscular diseases, now classified as chronic progressive external ophthalmopelia (CPEO) and the Kearns-Sayre Syndrome (KSS), which result from mtDNA deletions (7) (Fig. 2). In the 10 years since these discoveries, over 50 pathogenic mtDNA base substitution mutations and hundreds of mtDNA rearrangement mutations (deletions and insertions) have been identified in a variety of degenerative diseases (8). Other mitochondrial diseases have been linked to nuclear genes whose inactivation either inhibits mitochondrial bioenergetics or disrupts mitochondrial or mtDNA biogenesis.

Here I review our current understanding of the genetics of mitochondrial disease, a

The author is at the Center for Molecular Medicine, Emory University, 1462 Clifton Road, Suite 420, Atlanta, GA 30322, USA. E-mail: dwallace@gmm.gen. emory.edu knowledge base derived primarily from patient studies, and then describe new mouse models that are providing important insights into pathophysiology.

\section{Mitochondrial Genetics}

Mitochondria generate cellular energy in the form of ATP (adenosine triphosphate) by the process of oxidative phosphorylation (OXPHOS). Most cells contain hundreds of mitochondria. These cytoplasmic organelles are thought to have arisen about 1.5 billion years ago from a symbiotic association between a glyolytic proto-eukaryotic cell and an oxidative bacterium. Modern mitochondria retain a num- ber of features that reflect their endosymbiotic origin. These include a double membrane structure and a circular mitochondrial genome with mitochondria-specific transcription, translation, and protein assembly systems. However, the mitochondrion has also adapted to its intracellular niche. To increase its replication rate, and thus ensure transmission to the two daughter cells at cytokinesis, the mammalian mitochondrial genome has been reduced in size to about 16,500 base pairs $(9-11)$. Presumably, this was accomplished by the deletion of nonessential genes and the transfer of many essential genes to the nucleus where the proteins are now transcribed into mRNAs, translated on cytoplasmic ribosomes, and selectively imported back into the mitochondrion (12).

The modern mammalian mtDNA retains only 13 polypeptide genes, all of which encode essential components of OXPHOS. It also encodes the $12 S$ and $16 S$ rRNA genes and the 22 tRNA genes required for mitochondrial protein synthesis (Fig. 2). The remaining mitochondrial OXPHOS proteins, the metabolic enzymes, the DNA and RNA polymerases, the ribosomal proteins, and the mtDNA regulatory factors, such as mitochondrial transcription factor A
Fig. 1. Mitochondrial myopathy in man and mouse. $(\mathbf{A})$ and $(\mathbf{C})$ are skeletal muscle samples from a patient with myoclonic epilepsy and ragged-red fiber disease (MERRF), which is caused by a mutation in the mitochondrially encoded tRNA ${ }^{\text {Lys }}$ gene $(38,39)$. (B) and (D) are skeletal muscle samples from a mouse with mitochondrial myopathy and hypertrophic cardiomyopathy resulting from the targeted inactivation of the gene encoding the heart-muscle isoform of the adenine nucleotide translocator (Ant1) (84). Frozen sections showing a single fiber (A) or several fibers (B) were stained with Gomori modified trichrome to show the ragged-red muscle fibers (RRFs). Electron micrographs show (C) an abnormal mitochondrion with paracrystalline arrays in a human RRF, and (D) the abnormal proliferation of mitochondria and degeneration of the contractile elements in a mouse RRF. 
(Tfam) are all encoded by nuclear genes (Fig. 2) $(4,5)$. The transfer of mtDNA sequences to the nucleus continues to this day $(13,14)$. However, as the mitochondrial genome became smaller, its genetic code began to drift. Today's mtDNA genes are no longer "intelligible" to the nucleocytosolic system and the mammalian mitochondrial genome is functionally stable (9).

The hundreds of mitochondria (and thousands of mtDNAs) within each cell's cytoplasm are transmitted through the oocyte's cytoplasm at fertilization and thus are strictly maternally inherited (15). The semiautonomous nature of the mitochondria and mtDNA has been demonstrated in experiments with cultured cells. A mtDNA mutation imparting resistance to the mitochondrial ribosome inhibitor chloramphenicol (CAP) has been transferred from CAPresistant $\left(\mathrm{CAP}^{\mathrm{R}}\right)$ cells to $\mathrm{CAP}$-sensitive $\left(\mathrm{CAP}^{\mathrm{S}}\right)$ cells by fusion of enucleated cytoplasmic fragments (cytoplasts) from the donor cell to the recipient cells $(16-18)$. This cytoplasmic hybrid or "cybrid" transfer technique is now a standard assay for determining whether cellular defects associated with mitochondrial disease are due to mtDNA mutations $(19,20)$.

Mitochondrial DNA has a very high mutation rate. When a mutation arises, cells initially contain a mixture of wild-type and mutant mtDNAs, a state known as heteroplasmy. During division of a heteroplasmic cell, the mutant and wild-type mtDNAs are randomly distributed into the daughter cells, such that over many generations the mtDNA genotype of a cellular lineage can drift toward predominantly mutant or wild-type mtDNAs (homoplasmy), a process known as replicative segregation. As the percentage of mutant mtDNAs increases, the cellular energy capacity declines until it falls below the bioenergetic threshold, the minimum energy output necessary for a cell or tissue to function normally. Beyond this point disease symptoms appear and become progressively worse $(4,5)$.

The stochastic and quantitative nature of mtDNA genetics means that the inheritance and expression of heteroplasmic mutations are highly variable. Indeed, it has been repeatedly demonstrated that the same mtDNA mutation can produce markedly different symptoms among members of the same family, in association with the chance fluctuation in the percentage of mutant mtDNAs that each individual inherits. Mutations in nuclear genes can also affect OXPHOS, often resulting in Mendelian diseases with phenotypes similar to those caused by mtDNA mutations. Hence, the genetics of mitochondrial diseases and the associated issues this raises in the context of genetic counseling can be extremely perplexing $(4,5)$.

\section{Mitochondrial Biology}

The vagaries of mitochondrial genetics are made even more complex by the multiple cellular functions performed by the mitochondri- on. Three of the more important aspects of mitochondrial OXPHOS for disease pathogenesis are: (i) energy production, (ii) generation of reactive oxygen species (ROS), and (iii) regulation of programmed cell death, or apoptosis.

The proteins involved in OXPHOS are located within the mitochondrial inner membrane and include the electron transport chain (ETC) components, ATP synthase, and the adenine nucleotide translocator (ANT) (Fig. 3). The ETC oxidizes hydrogen derived from the oxidation of organic acids such as pyruvate and fatty acids with atomic oxygen to generate water. The electrons, borne on $\mathrm{NAD}^{+}$(nicotinamide adenine dinucleotide), are transferred to respiratory complex I (NADH dehydrogenase) and then to coenzyme $\mathrm{Q}_{10}(\mathrm{CoQ})$, and the electrons from succinate in the tricarboxylic acid (TCA) cycle are transferred to complex II (succinate dehydrogenase, SDH) and to CoQ. From CoQ, the electrons are passed to complex III, then to cytochrome c (cyt c), then to complex IV (cytochrome c oxidase, COX), and finally to $1 / 2 \mathrm{O}_{2}$ to give $\mathrm{H}_{2} \mathrm{O}$. The energy released is used to pump protons $\left(\mathrm{H}^{+}\right)$out of the mitochondrial inner membrane to create an electrochemical gradient $(\Delta \Psi)$ that is positive and acidic on the outside and negative and alkaline on the mitochondrial matrix side. This creates a capacitor that can be depolarized by the transport of protons back into the matrix through a proton channel in the $\mathrm{F}_{0}$ mem- brane component of the ATP synthase. The proton flux drives the condensation of ADP (adenosine diphosphate) and $\mathrm{P}_{\mathrm{i}}$ (inorganic phosphate) to make ATP, which is then exported to the cytosol in exchange for the spent ADP by the ANT. In this way, oxygen consumption by the ETC is coupled to ADP phosphorylation by the ATP synthase through the electrochemical gradient, $\Delta \Psi(3-5)$.

OXPHOS is the major endogenous source of the $\operatorname{ROS}\left(\mathrm{O}_{2}{ }^{\cdot-}, \mathrm{H}_{2} \mathrm{O}_{2}\right.$, and $\left.\mathrm{OH}^{\cdot}\right)$, which are toxic by-products of respiration (Fig. 3). This is because when the ETC is inhibited, the electrons accumulate in the early stages of the ETC (complex I and CoQ), where they can be donated directly to molecular oxygen to give superoxide anion $\left(\mathrm{O}_{2}{ }^{-}\right)$. Superoxide anion is detoxified by the mitochondrial Mn superoxide dismutase (MnSOD) to give hydrogen peroxide $\left(\mathrm{H}_{2} \mathrm{O}_{2}\right)$, and $\mathrm{H}_{2} \mathrm{O}_{2}$ is converted to $\mathrm{H}_{2} \mathrm{O}$ by glutathione peroxidase (GPx). $\mathrm{H}_{2} \mathrm{O}_{2}$, in the presence of reduced transition metals, can also be converted to the highly reactive hydroxyl radical $\left(\mathrm{OH}^{\circ}\right)$ by the Fenton reaction. Chronic ROS exposure can result in oxidative damage to mitochondrial and cellular proteins, lipids, and nucleic acids, and acute ROS exposure can inactivate the ironsulfur (Fe-S) centers of ETC complexes I, II, and III, and TCA cycle aconitase, resulting in shutdown of mitochondrial energy production $(3,4)$.

Mitochondria also provide a major switch

Fig. 2. The human mtDNA map, showing the location of selected pathogenic mutations within the 16,569base pair genome. Human mtDNA codes for seven of the 43 subunits of complex I (ND1, 2, 3, 4, 4L, 5, and 6), shown in red; one of the 11 subunits of complex III (cytochrome b, cyt b), shown in orange; three of the 13 subunits of complex IV (COI, II, and III), shown in purple; and two of 16 subunits of complex $V$ (ATPase 6 and 8), shown in yellow. It also codes for the small and large rRNAs, shown in green; and 22 tRNAs, shown in beige, with the adjacent letters indicating the cognate amino acids. The heavy $(\mathrm{H})$-strand origin of replication $\left(\mathrm{O}_{\mathrm{H}}\right)$ and the $\mathrm{H}$-strand and light (L)strand promoters, $\mathrm{P}_{\mathrm{H}}$ and $P_{t}$, are indicated in the control region. The mitochon-

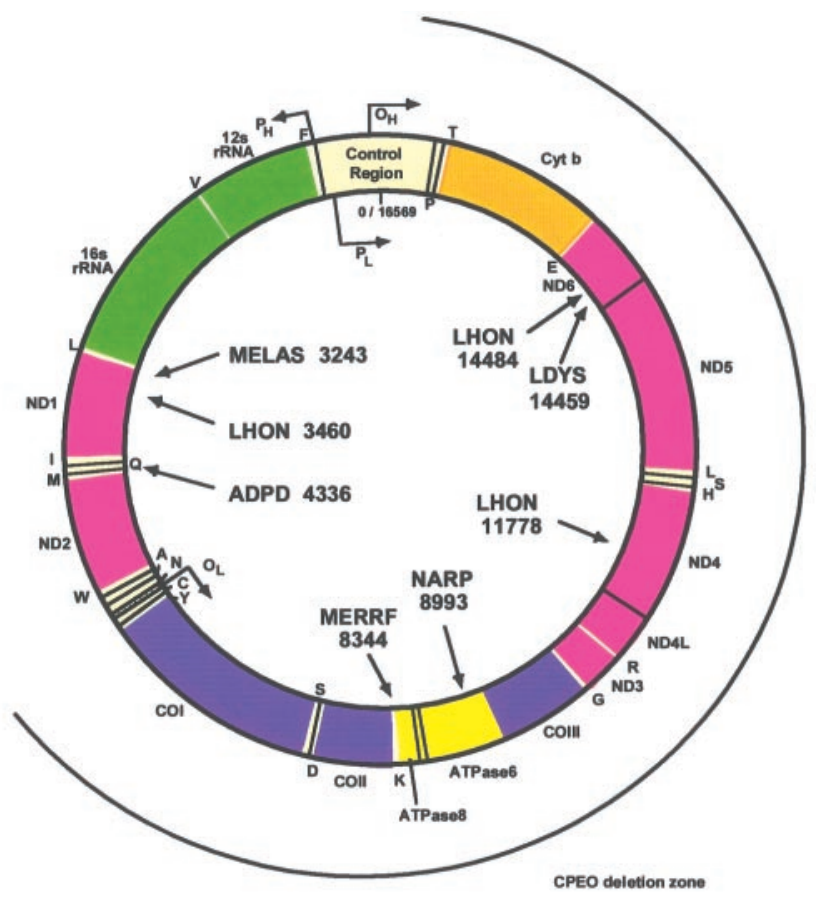
drial transcription factor

Tfam binds between the promoters. Tfam is important in initiating transcription and in generating the primer from the L-strand transcripts to initiate $\mathrm{H}$-strand DNA replication at $\mathrm{O}_{\mathrm{H}}$. The L-strand origin of replication $\left(\mathrm{O}_{\mathrm{L}}\right)$ is located two-thirds of the way around the genome. The positions of representative pathogenic point mutations are shown on the inside of the circle, with the nucleotide position and disease acronym. All acronyms are defined in the text except "DEAF," which signifies sensory neural deafness and "ADPD," which signifies late-onset Alzheimer's disease $(4,5,8)$. 
for the initiation of apoptosis. This switch is thought to involve the opening of a nonspecific mitochondrial inner membrane channel, the mitochondrial permeability transition pore (mtPTP) (Fig. 3) (21-23). The mitochondrial inner membrane space contains a number of cell death-promoting factors, including cyt c, apoptosis-inducing factor (AIF, a flavoprotein), and latent forms of specialized proteases called caspases. Opening of the mtPTP causes collapse of $\Delta \Psi$, swelling of the mitochondrial inner membrane, and release of these death-promoting factors. The cyt $\mathrm{c}$ activates the cytosolic caspase protein degradation pathway, leading to destruction of the cytoplasm. AIF translocates to the nucleus, inducing chromatin destruction (24, 25). Opening of the mtPTP and the accompanying death of the cell can be initiated by the mitochondrion's excessive uptake of $\mathrm{Ca}^{2+}$, increased exposure to ROS, or decline in energetic capacity (22-26). Thus, a marked reduction in mitochondrial energy production and a chronic increase in oxidative stress could theoretically activate the mtPTP and initiate apoptosis.

The interaction of these three OXPHOS processes may explain some of the perplexing pathophysiological features of mitochondrial disease. For example, mutations that disrupt mitochondrial OXPHOS would re- duce energy output and presumably impair multiple cellular processes. Inhibition of the ETC is likely to increase ROS production and oxidative stress. Increased oxidative stress and decreased energy levels might activate the mtPTP, leading to apoptosis. Consistent with this scenario, in patients with Complex I defects, the severity of the enzyme deficiency has been correlated with increased $\mathrm{O}_{2}{ }^{--}$production and induction of MnSOD mRNA (27). Moreover, in skeletal muscle samples from patients with CPEO and MELAS (mitochondrial encephalomyopathy, lactic acidosis, and stroke-like episodes), the RRFs showed localized increases in MnSOD protein (28).

\section{Mitochondrial Diseases}

As noted earlier, mitochondrial diseases can have a wide variety of inheritance patternsmaternal, Mendelian, and a combination of the two. Adding to this complexity is the fact that the phenotypes of mitochondrial diseases can be both diverse and overlapping; that is, the same mtDNA mutation can produce quite different phenotypes, and different mutations can produce similar phenotypes. Hence, for mitochondrial diseases it has been more productive to classify patients by genetic defect rather than by clinical manifestation.

Pathogenic mtDNA mutations include both base substitutions and rearrangement mutations $(8)$. The base substitutions can be subdivided into missense mutations that affect the 13 protein-encoding genes and those that affect the rRNA or tRNA genes, which have global effects on mitochondrial protein synthesis $(4,5)$.

The mtDNA mutations that cause LHON, dystonia, and Leigh's disease provide good examples of the clinical variability that can accompany a heteroplasmic missense mutation. LHON and dystonia can be caused by the same MTND6*LDYT14459A mutation, a G to A transition in the mitochondrial ND6 gene, which encodes a subunit of NADH dehydrogenase. The mutation converts a highly conserved alanine at codon 72 to a valine. Patients with LHON present in midlife with sudden-onset blindness caused by death of the optic nerve. Patients with dystonia present early in life with a generalized movement disorder, impaired speech, mental retardation, and short stature, frequently accompanied by degeneration of the brain's basal ganglia. LHON is thought to be associated with a lower percentage of mutant mtDNA and dystonia with a higher percentage (29-31). The MTND6*LDYT14459A mutation causes a substantial reduction in cellular complex I activity and this defect can be transferred between cells in cybrid fusions (32). However, it is not clear why the same biochem-

Fig. 3. Diagram of a mitochondrion, illustrating the relationships between mitochondrial oxidative phosphorylation and (i) the production of energy (ATP), (ii) the generation of reactive oxygen species (ROS), and (iii) the initiation of apoptosis through activation of the mitochondrial permeability transition pore (mtPTP). The respiratory enzyme complexes involved in OXPHOS are complex I [NADH: ubiquinone oxidoreductase], which includes a flavin mononucleotide and six $\mathrm{Fe}-\mathrm{S}$ centers (designated with a cube); complex II [succinate: ubiquinone oxidoreductase], which includes a flavin-adenine dinucleotide, three Fe-S centers, and a cytochrome b; complex III [ubiquinol: cytochrome c oxidoreductase], which includes cytochrome b, cytochrome $\mathrm{c} 1$, and the Rieske Fe-S center; complex IV [cytochrome c oxidase], which includes cytochromes $a+a_{3}, \mathrm{CuA}$ and $\mathrm{CuB}$; and complex $\mathrm{V}\left[\mathrm{H}^{+}\right.$-translocating ATP synthase]. Pyruvate from glucose enters the mitochondria via pyruvate dehydrogenase $(\mathrm{PDH})$, generating acetyCoA, which enters the tricarboxylic acid (TCA) cycle by combining with oxaloacetate (OAA). cis-Aconitase converts citrate to isocitrate and contains an $4 \mathrm{Fe}-4 \mathrm{~S}$ center. Lactate dehydrogenase (LDH) converts excess pyruvate plus NADH to lactate (3-5). Small molecules diffuse through the outer membrane via the voltagedependent anion channel (VDAC) or porin. VDAC together with the

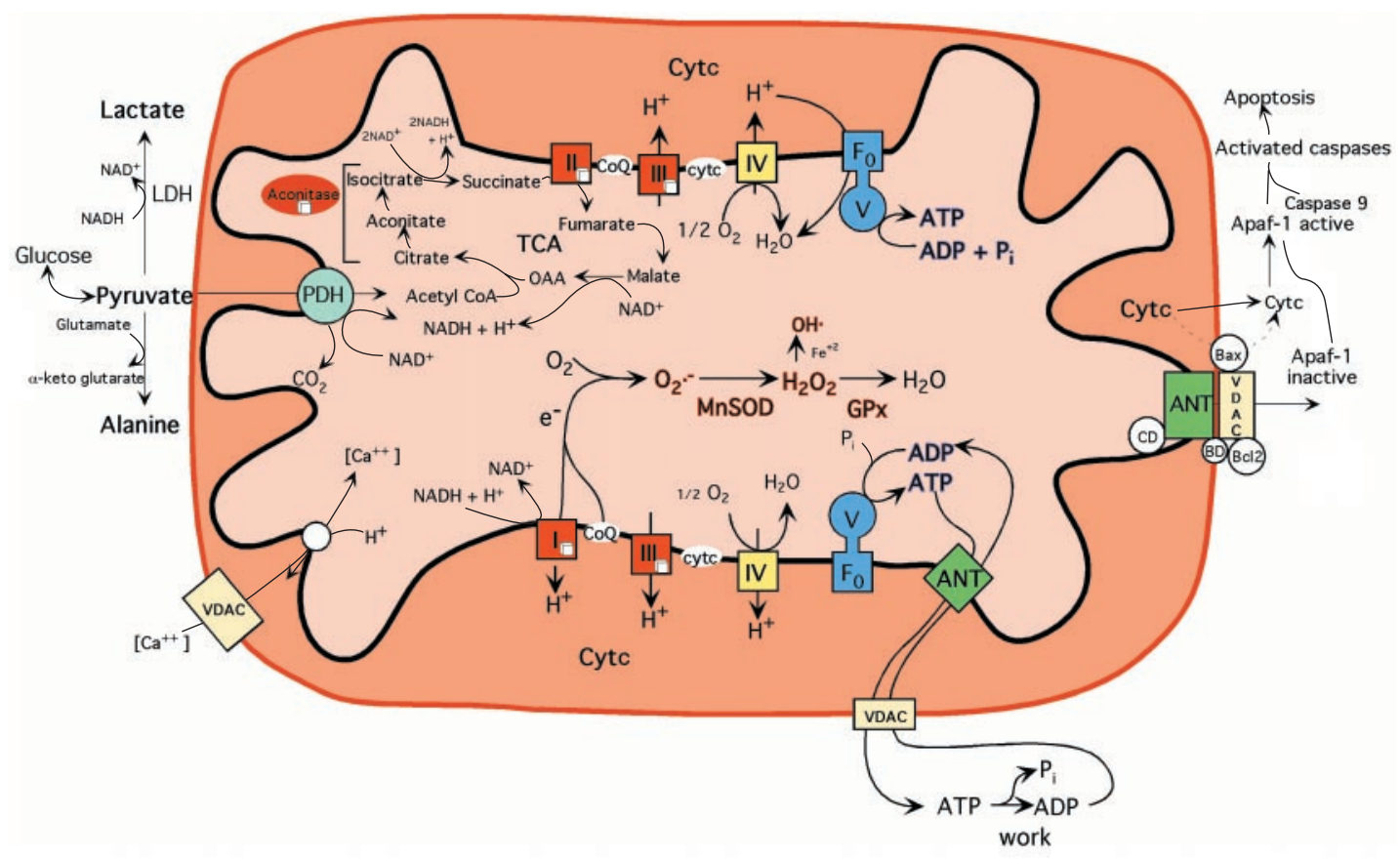

adenine nucleotide translocator (ANT), Bax, and cyclophilin D (CD) are thought to come together at the mitochondrial inner and outer membrane contact points to create the $\operatorname{mtPTP}(22,23,26)$. Bax is pro-apoptotic and is thought to interact with the anti-apoptotic protein $\mathrm{BCl} 2$ and the benzodiazepine receptor (BD). The opening of the mtPTP is associated with the release of AIF and cyt $c$, and activation by cyt c of Apaf- 1 and pro-caspase- 9 . The activated caspase- 9 then initiates the proteolytic degradation of cellular proteins, leading to cell death (21-25). 
ical defect can result in such different clinical phenotypes.

Leigh's syndrome can be caused by the MTATP6*NARP8993G mutation, a T to G transition in the ATP6 gene (encoding subunit 6 of mitochondrial ATP synthase) that changes the conserved leucine at codon 156 to an arginine (33). This mutation is invariably heteroplasmic and, when present in a small percentage $(<75 \%)$ of mtDNAs, it can cause neurogenic muscle weakness, ataxia, and retinitus pigmentosa (NARP). However, when present in a higher percentage $(>95 \%)$ of mtDNAs, it can cause Leigh's syndrome (34-36), an early-onset disease that is frequently lethal. Leigh's syndrome is associated with ataxia, hypotonia, spasticity, developmental delay, optic atrophy, ophthalmoplegia (paralysis of the extra-ocular eye muscles), developmental delay, and subsequent regression. The end-stage disease is generally associated with degeneration of the basal ganglia accompanied by vascular proliferation (5). The MTATP6*NARP8993G mutation causes a block in the ATP synthase $F_{O}$ proton channel, a defect that can be transferred in cybrid experiments (37). Thus, variation in the percentage of mutant mtDNAs between patients must change the ATP output and cause the variation in clinical symptoms.

Mutations in mitochondrial protein synthesis genes can produce a complex array of symptoms. In severe cases the mutations are heteroplasmic and frequently cause central nervous system (CNS) abnormalities as well as mitochondrial myopathy with ragged-red fibers (RRFs) (Fig. 1) (2-5), an association referred to as mitochondrial encephalomyopathy. CNS manifestations can include sensory neural hearing loss, epilepsy, stroke-like episodes, and progressive dementia. Cardiomyopathy, lactic acidosis, and endocrine disorders, including diabetes mellitus, are also common (4). Although the mitochondrial encephalomyopathies frequently share certain clinical features such as RRFs, specific mutations are often associated with specific clinical manifestations. The MTTK*MERRF8344G mutation in the tRNA $^{\text {Lys }}$ gene can cause myoclonic epilepsy and RRFs (hence the acronym MERRF) (38, 39). The MTTL1*MELAS3243G mutation in the tRNA ${ }^{\text {Leu }}$ gene is often associated with stroke-like activity and mitochondrial myopathy (MELAS) when it is present in a high percentage $(>85 \%)$ of mtDNAs (40), but it is associated with maternally inherited diabetes mellitus and deafness when present in a low percentage (5 to $30 \%)$ of mtDNAs $(41,42)$. Cultured cells from patients harboring either of these mutations have reduced levels of mitochondrial protein synthesis and complex I and IV activities, and these defects can be transferred along with the mutant mtDNA in cybrid transfer experiments (43-45). Milder mitochondrial protein synthesis mutations can be homoplasmic and only affect the CNS. One example is the MTTQ*ADPD4336C mutation in the $\mathrm{tRNA}^{\mathrm{Gln}}$ gene, which is consistently homoplasmic and has been associated with lateonset Alzheimer's disease $(46,47)$.

Diseases resulting from mtDNA rearrangements include CPEO and KSS, maternally inherited diabetes mellitus and deafness (48, 49), and the spontaneously occurring Pearson's marrow-pancreas syndrome, a fatal disease associated with pediatric pancytopenia (loss of all blood cells) (50). CPEO and the KSS patients present with mitochondrial myopathy including ophthalmoplegia and ptosis (droopy eyelids), and a subset have cardiac defects, renal problems, diabetes mellitus, and other symptoms. Histological analysis of CPEO and KSS muscle has revealed bands of COX-deficient $\left(\mathrm{COX}^{-}\right)$and $\mathrm{SDH}-$ hyperreactive $\left(\mathrm{SDH}^{+}\right)$activity along the muscle fibers. These $\mathrm{COX}^{-}$and $\mathrm{SDH}^{+}$fibers generally correspond to regions where there is mitochondrial proliferation with RRFs, high levels of mutant mtDNA, and induced expression of OXPHOS genes (51-54).

Mitochondrial DNA diseases commonly have a delayed onset and a progressive course. This implies that the phenotypic expression of these diseases may involve two factors, the predisposing mutation and an age-related factor that causes a decline in mitochondrial function, which exacerbates the inherited defect (4).

Mitochondrial diseases resulting from mutations in nuclear OXPHOS genes exhibit Mendelian inheritance patterns, yet share many of the clinical features of mtDNA mutations. For example, a mutation in the gene encoding the $18-\mathrm{kD}$ structural protein of complex I has been reported in a child manifesting hypotonia, mental retardation, convulsions, and basal ganglia degeneration (55). A mutation in a mitochondrial protease-like adenosine triphosphatase (ATPase) has been associated with autosomal dominant spastic paraplegia (56). Mutations in a number of nuclear genes have been associated with Leigh's syndrome. These include the genes encoding the E1 $\alpha$ subunit of pyruvate dehydrogenase (57), the NDUF8 N-2 Fe-S center protein of complex I (58), the flavoprotein subunit of complex II (59), and the SURF-1 protein associated with complex IV defects $(60,61)$.

Mutations in nuclear genes can also exert their phenotypic effects by indirectly inactivating OXPHOS or destabilizing the mtDNA. Friedreich's ataxia is an autosomal recessive disease that results in cerebellar ataxia, peripheral neuropathy, and hypertrophic cardiomyopathy. The mutant protein, frataxin, is targeted to the mitochondrial inner membrane and functions to transport iron out of the mitochondrion. With the loss of this protein, iron accumulates in the mitochondrial matrix, stimulating the conversion of $\mathrm{H}_{2} \mathrm{O}_{2}$ to $\mathrm{OH}^{-}$by the Fenton reaction. This inactivates the mitochondrial Fe-S center enzymes (complexes I, II, III, and aconitase), which in turn reduces mitochondrial energy production (62).

Other diseases that arise from nucleocytoplasmic interactions include the autosomal dominant-progressive external ophthalmoplegia (AD-PEO), the mtDNA depletion syndrome, and the MNGIE syndrome. AD-PEO families inherit a dominant nuclear mutation that predisposes them to accumulate multiple mtDNA deletions in their skeletal muscle, and to develop mitochondrial myopathy and PEO. The disease has been linked to two different chromosomal loci, but the responsible genes have not been identified $(63,64)$. The mtDNA depletion syndrome is associated with severe reductions in the mtDNA levels of muscle, liver, or kidney, resulting in organ failure and death. This syndrome is likely caused by a nuclear mutation that disrupts the regulation of mtDNA copy number during development, resulting in random loss of the mtDNA $(65,66)$.

The mitochondrial neurogastrointestinal encephalomyopathy (MNGIE) syndrome is associated with mitochondrial myopathy with RRFs and abnormal mitochondria, decreased respiratory chain activity, and multiple mtDNA abnormalities. This autosomal recessive disease has been linked to mutations in $T P$, a nuclear gene encoding thymidine phosphorylase, although the clinical symptoms probably result from the destruction of the mtDNA. It has been hypothesized that inactivation of TP alters cellular thymidine pools that are important in mtDNA maintenance (67).

\section{Somatic mtDNA Mutations in Aging and Cancer}

The delayed onset and progressive course of mitochondrial diseases suggests that mitochondria function may decline with age. This hypothesis is supported by multiple reports of age-related declines in primate mitochondrial OXPHOS enzyme activities in skeletal muscle, liver, and brain $(3,68)$, and the associated accumulation of somatic mtDNA rearrangements in these same postmitotic tissues. For example, polymerase chain reaction (PCR) experiments have shown that skeletal muscle from human subjects under the age of 40 contains primarily intact mtDNAs, whereas skeletal muscle from subjects over the age of 50 shows an accumulation of a wide array of mtDNA rearrangements (69). In addition, the skeletal muscle of elderly subjects has been found to have RRFs, with each $\mathrm{COX}^{-}$and $\mathrm{SDH}^{+}$fiber containing a different mtDNA mutation $(3,70)$. This confirms that each of the mutations arose de novo and was selectively amplified within the cell to create the 
regional respiratory defects.

Somatic mtDNA mutations also occur in the brain. Quantitation of the common 5-kb mtDNA deletion has shown that mtDNA deletions accumulate markedly in the basal ganglia and various cortical regions in humans after age $75(71,72)$. An analogous agerelated accumulation of somatic mtDNA rearrangements also occurs in mouse tissues (73), the extent of which is proportional to life-span rather than absolute time.

The cause of the somatic mtDNA mutations is likely to be oxidative damage, which increases with age in the mtDNA of both man and mouse $(3,74,75)$. Patients with chronic ischemic heart disease, which is associated with cyclic bursts of mitochondrial ROS during ischemia and reperfusion (76), have been found to harbor 8 to 2000 times more mtDNA deletions in the heart than age-matched controls (77). Similarly, cortical mtDNA deletion levels are elevated in patients with Alzheimer's and Huntington's disease $(78,79)$, and mtDNA from the former group shows increased oxidative damage (80).

These observations have led to the hypothesis that somatic mtDNA mutations accumulate in postmitotic tissues with age as a result of mitochondrial ROS damage. The resulting agerelated decline in OXPHOS would ultimately degrade the tissue's bioenergetic capacity until it falls below a certain threshold, resulting in symptoms and senescence. This same agerelated decline in OXPHOS could interact with inherited mitochondrial defects, which would account for the delayed onset and progression of mitochondrial diseases.

Somatic mtDNA mutations have also been identified in various tumors and tumor cell lines. These mutations include intragenic deletions (81), missense and chain-termination point mutations (82), and alterations of homopolymeric sequences that result in frameshift mutations (83). In principle, these mutations could contribute to neoplastic transformation by changing cellular energy capacities, increasing mitochondrial oxidative stress, and/or modulating apoptosis.

\section{Mouse Models of Mitochondrial Disease}

Patient studies have revealed much about the genetics of mitochondrial disease, but the pathophysiological mechanisms that underlie the complex array of symptoms remain mysterious. Recently, new insights into this question have been obtained by the creation and analysis of mouse models for mitochondrial disease. Four such models are discussed below.

Ant1-deficient mice. Targeted inactivation of the nuclear-encoded Ant1 gene has provided a model for chronic ATP deficiency. Surprisingly, ANT1-deficient (Ant1 ${ }^{-/-}$) mice are viable, although they develop classical mitochondrial myopathy and hypertrophic cardiomyopathy (84).

In mice, ANT is encoded by two genes. Ant1 is expressed at high levels in skeletal muscle and heart and at lower levels in brain, whereas Ant2 is expressed in all tissues but skeletal muscle (84). Thus, Ant1 ${ }^{-/-}$mice are completely deficient in ANT in skeletal muscle, partially deficient in heart, and have normal ANT levels in liver. The skeletal muscle of Ant1 ${ }^{-/-}$mice exhibit classic RRFs (Fig. 1) and increased SDH and COX staining in the Type I oxidative muscle fibers. These elevated OXPHOS enzyme activities correlate with a massive proliferation of giant mitochondria in the skeletal muscle fibers, degeneration of the contractile fibers (Fig. 1), and a marked exercise fatigability. The hypertrophic cardiomyopathy is also associated with mitochondrial proliferation.

Both nuclear and mitochondrial OXPHOS genes are upregulated in $\mathrm{Ant}^{-1-}$ mice. Among the transcripts overexpressed in skeletal muscle are mRNAs for several components of complex I and complex IV, various mitochondrial transcripts, and the mRNA for Mcl-1, a muscle homolog of Bcl-2 (Fig. 3) (85). This implies that ANT1 deficiency may affect not only mitochondrial bioenergetics, but also oxidative stress and possibly apoptosis.

Ant $1^{-1-}$ mice also have elevated serum levels of lactate, alanine, and succinate, consistent with inhibition of the respiratory chain and TCA cycle. Their skeletal muscle mitochondria are completely resistant and their heart mitochondria are partially resistant to the stimulation of oxygen consumption by exogenous ADP. This is consistent with the reductions in ANT levels and the associated inhibition of ADP/ATP exchange. The resulting limitation of available matrix ADP for the ATP synthase would be expected to reduce proton flux through its $\mathrm{F}_{\mathrm{o}}$ proton channel, hyperpolarize the mitochondrial inner membrane inhibiting the ETC, and redirect electrons from the ETC into the ROS-generating pathway. Indeed, $\mathrm{H}_{2} \mathrm{O}_{2}$ production was increased six- to eightfold in skeletal muscle and heart mitochondria from Ant $1^{-/}$mice, to levels comparable to those observed when control mitochondria are treated with the complex III inhibitor Antimycin A. This increase in ROS production was paralleled by an eightfold induction of the $\mathrm{O}_{2}^{--}$detoxifying enzyme MnSOD in skeletal muscle mitochondria, but not in heart mitochondria (86). In the heart, the increased $\mathrm{O}_{2}{ }^{--}$production, without a compensating induction in MnSOD, was associated with a dramatic increase in heart mtDNA damage. In fact, the heart mtDNAs of 16- to 20-month-old Ant1 ${ }^{-1-}$ mice had mtDNA rearrangement levels comparable to those seen in 32-monthold Ant $1^{+/+}$animals (86). Hence, inhibition of OXPHOS not only reduces energy produc- tion, but also elevates ROS production with an associated increase in damage to the mitochondria and mtDNA.

MnSOD-deficient mice. The origins and consequences of increased mitochondrial ROS production have been confirmed by studying mice carrying inactivating mutations in the nuclear gene encoding mitochondrial MnSOD. Two mouse lines lacking MnSOD (Sod2) have been constructed, Sod2 $2^{\mathrm{tm} 1 \mathrm{Cje}}(87)$ and Sod2 $2^{\mathrm{tm} 1 \mathrm{Leb}}(88)$. The Sod $2^{\mathrm{tm} 1 \mathrm{Cje}}$ mutation on the CD1 background results in neonatal death from dilated cardiomyopathy (87). The Sod2 $2^{\text {tm1Leb }}$ mutation on the C57BL/6 background results in death at about day 18 , in association with neuronal degeneration in the basal ganglia and brain stem (88).

The Sod2 ${ }^{\mathrm{tm} 1 \mathrm{Cj} e}$ mutation on the CD1 background has been extensively characterized. These MnSOD-deficient mice die from dilated cardiomyopathy at about 8 days of age $(87,89)$, and they exhibit striking lipid deposits in the liver. The mice also have significant reductions of complex II and complex III activity in skeletal muscle and heart, of complex I activity in heart, and of mitochondrial aconitase activity in heart and brain. In addition, the animals' urine contains organic acids that are characteristically found in the urine of patients with HMG (3-hydroxy-3-methylglutaryl)-CoA lyase deficiency, and the activity of this enzyme is significantly reduced in the liver of the mutant mice. Finally, DNA from the heart and brain of the mice shows accumulation of oxidative DNA damage (90).

These observations indicate that the high levels of mitochondrial $\mathrm{O}_{2}^{--}$anion resulting from the MnSOD-deficiency can inhibit the ETC (complexes I and II) and the TCA cycle (aconitase), probably by inactivating the $\mathrm{Fe}-\mathrm{S}$ centers in these enzymes. Consistent with this notion, the cardiac defect in the Sod $2^{-/-}$mice can be rescued by administration of the antioxidant MnTBAP [manganese 5,10,15,20-tetrakis (4-benzoic acid) porphyrin], which converts $\mathrm{O}_{2}{ }^{--}$to $\mathrm{H}_{2} \mathrm{O}_{2}(89)$. Peritoneal injection of this compound eliminated the dilated cardiomyopathy, reduced the liver lipid deposition, and extended the mean life span of the mice from 8 to 16 days. However, MnTBAP does not cross the blood-brain barrier, and by 12 days of age the MnTBAP-treated mice began to develop debilitating movement disorders in association with neuronal cell loss in the brain's motor centers.

Although the acute pathology observed in the Sod2 $2^{-1-}$ mice confirms the importance of mitochondrial ROS toxicity in mitochondrial dysfunction, many clinical syndromes such as Friedreich's ataxia are associated with chronic ROS exposure. The analysis of heterozygous Sod2 $2^{+/-}$mice (91), which exhibit a $50 \%$ reduction in MnSOD levels, may pro- 
vide a better model for chronic mitochondrial disease. The liver mitochondria of these mice have a $30 \%$ reduction in glutathione levels, aconitase activity, and complex I activity. The mice also show signs of oxidative damage to mitochondrial DNA and protein. Finally, there was a reduction in ADP-stimulated respiration, indicating partial uncoupling of the mitochondria, and the mitochondria have increased sensitivity to calcium and oxidative stress-induced opening of the mtPTP (91). These results confirm that chronic exposure to mitochondrial ROS impairs mitochondrial function and possibly potentiates apoptosis.

Tfam-deficient mice. Inactivation of the nuclear gene encoding mitochondrial transcription factor Tfam may provide a model for the mtDNA deletion syndrome. The Tfam gene was inactivated in all tissues (92) by bracketing the terminal two exons with loxP sites, and eliminating these exons by crossing the $+/$ Tfam $^{\text {loxp }}$ mice with mice bearing the Cre recombinase gene under control of the $\beta$-actin gene promoter. The $\mathrm{Tfam}^{+/-}$mice were viable and reproductively competent, and had a 50\% reduction in Tfam transcript and protein levels, a $34 \%$ reduction in mtDNA copy number, a $22 \%$ reduction in mitochondrial transcript levels, and a partial reduction in COX protein levels in heart but not liver. The $\mathrm{Tfam}^{-1-}$ mice died between embryonic days E8.5 and E10.5, and showed a complete absence of Tfam protein and little detectable mtDNA. The mitochondria in the $\mathrm{Tfam}^{-1-}$ mice were enlarged with abnormal cristae and were deficient in COX but not SDH (92).

Mice homozygous for the Tfam $^{\text {loxP }}$ allele were then crossed with mice carrying the Cre recombinase gene driven by the muscle creatinine kinase gene promoter. This resulted in the selective ablation of Tfam function in heart and skeletal muscle (93). The hearts of 18.5-day mutant embryos had reduced levels of Tfam, but were otherwise morphologically and biochemically normal. However, the mutant mice died about 3 weeks after birth of dilated cardiomyopathy. Under anaesthesia, the mice developed atrioventricular conduction blocks. They also showed reduced levels of Tfam protein and mtDNA transcripts in heart and muscle, a reduced level of heart and skeletal muscle mtDNA, and reduced activity of respiratory complexes I and IV. Finally, some of the cardiomyocytes were $\mathrm{COX}^{-}$and $\mathrm{SDH}^{+}$, comparable to the mosaic OXPHOS deficiencies seen in human mitochondrial myopathy patients (93). Hence, these animals exhibit many of the features seen in the CPED mtDNA deletion and mtDNA deletion syndromes.

$C A P^{R}$ mice? With the goal of creating a model that accurately reflects the genetic complexity of mtDNA diseases, efforts are being made to introduce deleterious mtDNA mutations into the mouse female germline.

Cytoplasts collected from mouse oocytes harboring one mtDNA type (for example, NZB/BINJ) have been fused with oocytes harboring a different mtDNA type (for example, C57BL/6 or BALB/c) (94-96). Alternatively, membrane-bound karyoplasts containing a zygote nucleus and a portion of the oocyte cytoplasm have been fused to enucleated eggs $(96,97)$. In both cases heteroplasmic mice have been obtained and used to study the kinetics of replicative segregation. While neither technique permits the introduction of deleterious mtDNA mutations, these studies revealed that certain tissues show directional mtDNA segregation with age, with the BALB mtDNA coming to predominate in blood and spleen and the NZB mtDNA in liver and kidney (95).

In another strategy, intact mitochondria have been isolated from the livers of Mus spretus mice and microinjected into Mus musculus domesticus oocytes. All of the 23 surviving blastocysts analyzed had detectable levels of $M$. spretus mtDNA (98).

Finally, the $\mathrm{CAP}^{\mathrm{R}}$ mtDNA mutation has been introduced into mouse female embryonic stem (ES) cells by cybrid transfer, with subsequent injection of the mutant ES cells into blastocysts, and generation of chimeric females. Introduction of $\mathrm{CAP}^{\mathrm{R}} \mathrm{mtDNAs}$ into mice may produce a good model of the human mitochondrial protein synthesis diseases, since this mutation alters mitochondrial ribosome function, resulting in decreased complex I and IV activities (99).

The first attempt to introduce $\mathrm{CAP}^{\mathrm{R}}$ mtDNA into mice was made in 1978 (100). $\mathrm{CAP}^{\mathrm{R}} \mathrm{B} 16$ melanoma cells were enucleated and fused to the teratocarcinoma cell line OTT6050, and the teratocarcinoma cybrids then injected into blastocysts. Five of the six chimeric mice were found by isozyme analysis to have 10 to $15 \%$ chimerism in various organs. However, there was no direct evidence that the $\mathrm{CAP}^{\mathrm{R}}$ mtDNA was present in the mice. More recently, $\mathrm{CAP}^{\mathrm{R}}$ mtDNAs from mouse 501-1 cells were transferred into the mouse female embryonic stem (ES) line AK11.1 (99). The 501-1 CAP ${ }^{\mathrm{R}}$ mutation is a point mutation in the $16 S$ rRNA gene (101) that creates a new restriction enzyme site that allows the foreign mtDNA to be tracked. In some cases, the ES cells were cured of their resident $\mathrm{CAP}^{\mathrm{S}}$ mitochondria, prior to fusion with the $\mathrm{CAP}^{\mathrm{R}}$ cytoplasts, resulting in nearly homoplasmic $\mathrm{CAP}^{\mathrm{R}} \mathrm{ES}$ cells. With these ES cells, 10 to $30 \%$ tissue chimerism was obtained in 20 mice. Three of five chimeric animals were positive for the $\mathrm{CAP}^{\mathrm{R}}$ mtDNA, with the highest levels found in the kidneys (20 to $50 \%$ of the total mtDNA) (99).

These early results suggest that it may soon be possible to introduce pathogenic mtDNA mutations, isolated in cultured cells, into the mouse germline. This technology would permit a direct investigation of how the stochastic fluctuation of heteroplasmic mtDNA mutations affect mitochondrial energy production, oxidative stress, and apoptosis. Such mouse models promise to recapitulate the genetic and physiological variation seen in mitochondrial diseases and may ultimately provide important new insights into the role of mitochondrial defects in a spectrum of progressive diseases, possibly including aging and cancer.

\section{References and Notes}

1. R. Luft, D. Ikkos, G. Palmieri, L. Ernster, B. A. Afzelius, J. Clin. Invest. 41, 1776 (1962).

2. S. DiMauro and E. Bonilla, in The Molecular and Genetic Basis of Neurological Disease, R. N. Rosenberg, S. B. Prusiner, S. DiMauro, R. L. Barchi, Eds. (Butterworth-Heinemann, Boston, 1997), pp. 201235.

3. D. C. Wallace, in (2), pp. 237-269.

4. M. D. Brown, M. T. Lott, in Emery and Rimoin's Principles and Practice of Medical Genetics, D. L. Rimoin, J. M. Connor, R. E. Pyeritz, A. E. H. Emery, Eds. (Churchill Livingstone, London, 1996), vol. 1, pp. 277-332.

5. J. M. Shoffner and D. C. Wallace, in The Metabolic and Molecular Basis of Inherited Disease, C. R. Scriver, A. L. Beaudet, W. S. Sly, D. Valle, Eds. (McGraw-Hill, New York, 1995), pp. 1535-1609.

6. D. C. Wallace et al., Science 242, 1427 (1988).

7. I. J. Holt, A. E. Harding, J. A. Morgan-Hughes, Nature 331, 717 (1988)

8. MITOMAP: A Human Mitochondrial Genome Database. Center for Molecular Medicine, Emory University. www.gen.emory.edu/mitomap.html

9. D. C. Wallace, Microbiol. Rev. 46, 208 (1982).

10. S. G. Andersson et al., Nature 396, 133 (1998).

11. B. F. Lang et al., ibid. 387, 493 (1997).

12. G. Schatz, J. Biol. Chem. 271, 31763 (1996).

13. D. C. Wallace, C. Stugard, D. Murdock, T. Schurr, M. D. Brown, Proc. Natl. Acad. Sci. U.S.A. 94, 14900 (1997).

14. M. Hirano et al., ibid., p. 14894.

15. R. E. Giles, H. Blanc, H. M. Cann, D. C. Wallace, ibid. 77, 6715 (1980)

16. C. L. Bunn, D. C. Wallace, J. M. Eisenstadt, ibid. 71, 1681 (1974).

17. D. C. Wallace, C. L. Bunn, J. M. Eisenstadt, J. Cell Biol. 67, 174 (1975)

18. D. C. Wallace, in Techniques in Somatic Cell Genetics, J. W. Shay, Ed. (Plenum Press, New York, 1982), vol. 12, pp. 159-187.

19. M. P. King and G. Attardi, Science 246, 500 (1989).

20. I. A. Trounce, Y. L. Kim, A. S. Jun, D. C. Wallace, Methods Enzymol. 264, 484 (1996).

21. P. X. Petit, S. A. Susin, N. Zamzami, B. Mignotte, G. Kroemer, FEBS Lett. 396, 7 (1996).

22. D. R. Green and J. C. Reed, Science 281, 1309 (1998).

23. M. Zoratti and I. Szabo, Biochim. Biophys. Acta. 1241, 139 (1995).

24. S. S. Susin et al., Nature 397, 441 (1999); W. C. Earnshaw, ibid., p. 387.

25. X. Liu, C. N. Kim, J. Yang, R. Jemmerson, X. Wang, Cell 86, 147 (1996)

26. N. Brustovetsky and M. Klingenberg, Biochemistry 35, 8483 (1996); I. Marzo et al., Science 281, 2027 (1998).

27. S. Pitkanen and B. H. Robinson, J. Clin. Invest. 98, 345 (1996)

28. N. Ohkoshi et al., Muscle Nerve 18, 1265 (1995).

29. E. J. Novotny et al., Neurology 36, 1053 (1986).

30. A. S. Jun, M. D. Brown, D. C. Wallace, Proc. Natl. Acad. Sci. U.S.A. 91, 6206 (1994).

31. J. M. Shoffner et al., Ann. Neurol. 38, 163 (1995).

32. A. S. Jun, I. A. Trounce, M. D. Brown, J. M. Shoffner, D. C. Wallace, Mol. Cell. Biol. 16, 771 (1996).

33. I. J. Holt, A. E. Harding, R. K. Petty, J. A. MorganHughes, Am. J. Hum. Genet. 46, 428 (1990). 
34. R. G. Ortiz et al., Arch. Ophthalmol. 111, 1525 (1993).

35. Y. Tatuch et al., Am. J. Hum. Genet. 50, 852 (1992). 36. J. M. Shoffner et al., Neurology 42, 2168 (1992).

37. I. Trounce, S. Neill, D. C. Wallace, Proc. Natl. Acad. Sci. U.S.A. 91, 8334 (1994).

38. D. C. Wallace et al., Cell 55, 601 (1988).

39. J. M. Shoffner et al., ibid. 61, 931 (1990)

40. Y. Goto, I. Nonaka, S. Horai, Nature 348, 651 (1990).

41. K. D. Gerbitz, J. M. van den Ouweland, J. A. Maassen, M. Jaksch, Biochim. Biophys. Acta. 1271, 253 (1995).

42. Y. Goto, Muscle Nerve 3, S107 (1995).

43. A. Chomyn et al., Mol. Cell. Biol. 11, 2236 (1991).

44. A. Chomyn et al., Proc. Natl. Acad. Sci. U.S.A. 89, 4221 (1992).

45. J. P. Masucci, M. Davidson, Y. Koga, E. A. Schon, M. P. King, Mol. Cell. Biol. 15, 2872 (1995).

46. J. M. Shoffner et al., Genomics 17, 171 (1993).

47. T. Hutchin and G. Cortopassi, Proc. Natl. Acad. Sci. U.S.A. 92, 6892 (1995)

48. S. W. Ballinger et al., Nature Genet. 1, 11 (1992).

49. S. W. Ballinger, J. M. Shoffner, S. Gebhart, D. A. Koontz, D. C. Wallace, ibid. 7, 458 (1994).

50. A. Rotig et al., Lancet i, 902 (1989).

51. A. Heddi, P. Lestienne, D. C. Wallace, G. Stepien, J. Biol. Chem. 268, 12156 (1993).

52. A. Heddi, P. Lestienne, D. C. Wallace, G. Stepien, Biochim. Biophys. Acta. 1226, 206 (1994).

53. S. Mita, B. Schmidt, E. A. Schon, S. DiMauro, E. Bonilla, Proc. Natl. Acad. Sci. U.S.A. 86, 9509 (1989).

54. E. A. Shoubridge, G. Karpati, K. E. M. Hastings, Cell 62, 43 (1990).

55. L. van den Heuvel et al., Am. J. Hum. Genet. 62, 262 (1998)

56. G. Casari et al., Cell 93, 973 (1998).

57. P. M. Matthews et al., Ann. Neurol. 33, 652 (1993).

58. J. Loeffen et al., Am. J. Hum. Genet. 63, 1598 (1998).

59. T. Bourgeron et al., Nature Genet. 11, 144 (1995).
60. V. Tiranti et al., Am. J. Hum. Genet. 63, 1609 (1998).

61. Z. Zhu et al., Nature Genet. 20, 337 (1998).

62. A. Rotig et al., ibid. 17, 215 (1997)

63. A. Suomalainen et al., ibid. 9, 146 (1995).

64. M. Zeviani et al., Biochim. Biophys. Acta. 1271, 153 (1995).

65. A. G. Bodnar, J. M. Cooper, I. J. Holt, J. V. Leonard A. H. Schapira, Am. J. Hum. Genet. 53, 663 (1993)

66. C. T. Moraes et al., ibid. 48, 492 (1991).

67. I. Nishino, A. Spinazzola, M. Hirano, Science $\mathbf{2 8 3}$ 689 (1999).

68. I. Trounce, E. Byrne, S. Marzuki, Lancet i, 637 (1989).

69. S. Melov, J. M. Shoffner, A. Kaufman, D. C. Wallace, Nucleic Acids Res. 23, 4122 (1995).

70. J. Muller-Hocker, K. Schneiderbanger, F. H. Stefani, B. Kadenbach, Mutat. Res. 275, 115 (1992).

71. M. Corral-Debrinski et al., Nature Genet. 2, 324 (1992).

72. N. W. Soong, D. R. Hinton, G. Cortopassi, N. Arnheim, ibid., p. 318.

73. S. Melov, D. Hinerfeld, L. Esposito, D. C. Wallace Nucleic Acids Res. 25, 974 (1997)

74. B. N. Ames, M. K. Shigenaga, T. M. Hagen, Proc. Natl. Acad. Sci. U.S.A. 90, 7915 (1993)

75. P. Mecocci et al., Ann. Neurol. 34, 609 (1993)

76. D. K. Das, A. George, X. K. Liu, P. S. Rao, Biochem Biophys. Res. Commun. 165, 1004 (1989).

77. M. Corral-Debrinski, J. M. Shoffner, M. T. Lott, D. C. Wallace, Mutat. Res. 275, 169 (1992).

78. M. Corral-Debrinski et al., Genomics 23, 471 (1994).

79. T. M. Horton et al., Neurology 45, 1879 (1995).

80. P. Mecocci, U. MacGarvey, M. F. Beal, Ann. Neurol. 36, 747 (1994).

81. T. M. Horton et al., Genes Chromosomes Cancer 15 95 (1996).

82. K. Polyak et al., Nature Genet. 20, 291 (1998).

83. W. Habano, S. Nakamura, T. Sugai, Oncogene 17, 1931 (1998)

84. B. Graham et al., Nature Genet. 16, 226 (1997).
85. D. Murdock, B. E. Boone, L. Esposito, D. C. Wallace, J. Biol. Chem., in press.

86. L. Esposito, S. Melov, A. Panov, D. C. Wallace, Proc. Natl. Acad. Sci. U.S.A., in press.

87. Y. Li et al., Nature Genet. 11, 376 (1995).

88. R. M. Lebovitz et al., Proc. Natl. Acad. Sci. U.S.A. 93, 9782 (1996).

89. S. Melov et al., Nature Genet. 18, 159 (1998).

90. S. Melov et al., Proc. Natl. Acad. Sci. U.S.A. 96, 846 (1999).

91. M. D. Williams et al., J. Biol. Chem. 273, 28510 (1998).

92. N. G. Larsson et al., Nature Genet. 18, 231 (1998).

93. J. Wang et al., ibid. 21, 133 (1999).

94. J. P. Jenuth, A. C. Peterson, K. Fu, E. A. Shoubridge, ibid. 14, 146 (1996).

95. J. P. Jenuth, A. C. Peterson, E. A. Shoubridge, ibid. 16 93 (1997).

96. F. V. Meirelles and L. C. Smith, Genetics 148, 877 (1998).

97. — ibid. 145, 445 (1997)

98. C. A. Pinkert, M. H. Irwin, L. W. Johnson, R. J. Moffatt, Transgenic Res. 6, 379 (1997).

99. S. Levy, K. Waymire, G. MacGregor, Y. Kim, D. C. Wallace, Transgenic Res., in press.

100. T. Watanabe, M. J. Dewey, B. Mintz, Proc. Natl. Acad. Sci. U.S.A. 75, 5113 (1978)

101. H. Blanc, C. T. Wright, M. J. Bibb, D. C. Wallace, D. A. Clayton, ibid. 78, 3789 (1981)

102. I thank my colleagues at the Center for Molecular Medicine who participated in this work. In alphabetical order, these include: J. Bilgar, M. Brown, R. Burke, P. Coskun, B. Cottrell, L. Esposito, P. Hatten-Mitchell, J. Kokoszak, S. Levy, M. Lott, G. MacGregor, S. Melov, D. Murdock, J. Petros, C. Stugard, J. Sligh, and K. Waymire. Supported by NIH grants HL45572, AG13154, and NS21328 and by a Johnson and Johnson Focused Giving Grant.

\title{
Oxidative Phosphorylation at the fin de siècle
}

\author{
Matti Saraste
}

Mitochondria produce most of the energy in animal cells by a process called oxidative phosphorylation. Electrons are passed along a series of respiratory enzyme complexes located in the inner mitochondrial membrane, and the energy released by this electron transfer is used to pump protons across the membrane. The resultant electrochemical gradient enables another complex, adenosine $5^{\prime}$-triphosphate (ATP) synthase, to synthesize the energy carrier ATP. Important new mechanistic insights into oxidative phosphorylation have emerged from recent three-dimensional structural analyses of ATP synthase and two of the respiratory enzyme complexes, cytochrome bc ${ }_{1}$ and cytochrome c oxidase. This work, and new enzymological studies of ATP synthase's unusual catalytic mechanism, are reviewed here.

Mitochondria generate most of the energy in animal cells. This occurs primarily through oxidative phosphorylation, a process in which electrons are passed along a series of carrier molecules called the electron transport chain. These electrons are generated from $\mathrm{NADH}$ (reduced nicotinamide adenine dinu-

The author is at the European Molecular Biology Laboratory, Meyerhofstrasse 1, Postfach 102209, D-69012, Heidelberg, Germany. E-mail Saraste@EMBLHeidelberg.de cleotide), which is produced by oxidation of nutrients such as glucose, and are ultimately transferred to molecular oxygen. The electron transport chain consists of four respiratory enzyme complexes arranged in a specific orientation in the mitochondrial inner membrane. The passage of electrons between these complexes releases energy that is stored in the form of a proton gradient across the membrane and is then used by ATP synthase to make ATP from ADP (adenosine 5' diphosphate) and phosphate (Fig. 1).
Our understanding of the basic principles of oxidative phosphorylation was greatly influenced by several landmark discoveries spanning nearly a century. ATP was discovered by Karl Lohmann in 1929 and its role in muscle contraction was established by Vladimir Engelhardt in 1934. Efraim Racker purified the catalytic component of the mitochondrial ATPase $\left(F_{1}\right.$ or factor 1$)$ in 1961, and in 1997, Paul Boyer and John Walker shared half of the Nobel Prize for the discovery that this enzyme functions in a novel way. Otto Warburg's characterization of "Atmungsferment," the respiratory enzyme, in 1924 established the phenomenon of cell respiration, to which ATP synthesis was linked by Herman Kaclkar in 1937. And perhaps most importantly, in 1961, Peter Mitchell proposed the general mechanistic principle of oxidative and photosynthetic phosphorylation (the chemiosmotic theory), which explains the coupling between respiration and ATP synthesis in mitochondria. This theory remained controversial until the mid-1970s, but is now a paradigm in the intellectual frame- 